Boise State University

ScholarWorks

Biology Faculty Publications and Presentations

Department of Biological Sciences

9-4-2008

\title{
Caspase-Cleaved TAR DNA Binding Protein-43 is a Major Pathological Finding in Alzheimer's Disease
}

Troy T. Rohn

Boise State University

Publication Information

Rohn, Troy T. (2008). "Caspase-Cleaved TAR DNA Binding Protein-43 is a Major Pathological Finding in Alzheimer's Disease". Brain Research, 1228, 189-198.

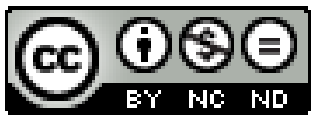

This is an author-produced, peer-reviewed version of this article. (C) 2009, Elsevier. Licensed under the Creative Commons AttributionNonCommercial-NoDerivatives 4.0 International License (https://creativecommons.org/licenses/by-nc-nd/4.0/). The final, definitive version of this document can be found online at Brain Research, doi:10.1016/j.brainres.2008.06.094 


\title{
Caspase-Cleaved TAR DNA Binding Protein-43 is a Major Pathological Finding in Alzheimer's Disease
}

\author{
Troy T. Rohn
}

\author{
${ }^{1}$ Department of Biology \\ Science/Nursing Building, Room 228 \\ Boise State University \\ Boise, Idaho, 83725 \\ Phone number: (208)-426-2396 \\ Fax number: $\quad(208-426-4267$ \\ Email address: trohn@boisestate.edu
}

Funded by NIH/NCRR grant \#P20RR016454 and a grant from the American Health Assistance Foundation (AHAF).

Key Words: Alzheimer's disease; TDP-43; Caspase; immunohistochemistry; immunofluorescence

The TAR DNA binding protein-43 (TDP-43) has been identified as a major constituent of inclusions found in frontotemporal dementia with ubiquitin-positive inclusions (FTLD-U) and amyotrophic lateral sclerosis (ALS). To determine a possible role for TDP-43 in Alzheimer's disease (AD), a site-directed caspase-cleavage antibody to TDP-43 based upon a known caspase-3 cleavage consensus site within TDP-43 at position D219 was designed. In vitro, this antibody labeled the predicted $25 \mathrm{kDa}$ caspase-cleavage fragment of TDP-43 without labeling full-length TDP-43 following digestion of recombinant TDP-43 with caspase-3 or treatment of Hela cells with staurosporine. Application of this antibody in postmortem brain sections indicated the presence of caspase-cleaved TDP-43 in Hirano bodies, tangles, reactive astrocytes and neuritic plaques of the AD brain. Caspase-cleaved TDP-43 also colocalized with ubiquitin labeled neurons as well as dystrophic neurites within plaque regions. These results suggest that caspase-cleaved TDP-43 is a major pathological finding in AD and may contribute to the neurodegeneration associated with this disease.

\section{Introduction}

The TAR DNA binding protein-43 (TDP-43) has been identified as a major disease protein in frontotemporal lobar degeneration with ubiquitin-positive inclusions (FTLD-U) and amyotrophic lateral sclerosis (ALS), \{Neumann, $2006 \# 2634$ \}. TDP-43 is a nuclear protein that is highly conserved and ubiquitously expressed in all tissues examined and whose function may be exon skipping and splicing inhibitory activity \{Buratti, 2004 \#2686\}. Other functions for TDP-43 include the biogenesis of mRNA and providing scaffolding for nuclear bodies \{Kwong, 2008 $\# 2685$ \}

In FTLD-U and ALS, TDP-43 immunoreactivity has been demonstrated within ubiquitin-positive neuronal cytoplasmic inclusions (NCIs), neuronal intranuclear inclusions, and neuronal dystrophic neurites \{Kwong, 2007 \#2639\}. In addition, recent studies have suggested that TDP-43 may also be a substrate for caspase-3 cleavage \{Zhang, 2007 \#2635\}, generating an approximate $25 \mathrm{kDa}$ fragment that has been identified in brain fractions from FTLD-U and ALS subjects \{Neumann, 2006 \#2634; Zhang, 2007 \#2635; Amador-Ortiz, 2007 \#2637\}. The presence of TDP-43 immunoreactivity has also been investigated in Alzheimer's disease (AD) with one study 
indicating a complete lack of TDP-43 labeling \{Josephs, 2008 \#2641\}, while other studies demonstrated TDP-43 within NCIs and a subset of neurofibrillary tangles (NFTs) in a minority of AD cases examined \{Amador-Ortiz, 2007 \#2637; Higashi, 2007 \#2642; Uryu, 2008 \#2688\}. Based upon these results, TDP-43 does not appear to be a major pathological feature associated with $\mathrm{AD}$.

In $\mathrm{AD}$, numerous studies have now demonstrated the activation of caspases and cleavage of cellular proteins including the amyloid precursor protein, fodrin, actin, GFAP and tau \{Gervais, 1999 \#717; Rohn, 2001 \#1798; Rohn, 2001 \#1799; Yang, 1998 \#2411; Mouser, 2006 \#2532; Rissman, 2004 \#2563; Cotman, 2005 \#2544\}. Therefore, we hypothesized that the lack of immunoreactivity to full-length TDP-43 antibodies in AD may reflect the fact that TDP-43 is cleaved by caspases and the epitopes to which full-length TDP-43 antibodies recognized is loss, thus explaining the apparent lack of labeling in the majority of $\mathrm{AD}$ cases examined. To test this hypothesis and to determine whether caspase-cleaved TDP-43 is present in the AD brain, we designed a site-directed caspase cleavage antibody to TDP-43 based upon a known caspase cleavage consensus site within TDP-43 at position 219 (DVMD) \{Zhang, 2007 \#2635\}. Application of this antibody in postmortem brain sections revealed widespread immunoreactivity in Hirano bodies, tangles, reactive astrocytes and within plaque-rich regions of all AD cases examined. Collectively, these results provide further support for the role of caspases in promoting the pathology associated with AD. Moreover, due to the extent and frequency of TDP-43 pathology uncovered, it is proposed that caspase-cleaved TDP-43 represents a major disease component associated with AD.

\section{Results}

Examination of the TDP-43 protein sequence indicates a putative caspase-cleavage consensus site, DVMD ${ }^{219}$, that would generate an amino-terminal fragment of approximately $25 \mathrm{kDa}$ following cleavage by caspases. We synthesized rabbit, polyclonal antibodies to this amino-terminal fragment within TDP-43 and tested their validity as specific probes for TDP-43 CCPs in a cell-free system or in a model system of apoptosis. Cell-free digestion of recombinant human TDP-43 with caspase-3 was undertaken and extracts were probed with the TDP-43 CCP (herein referred to as TDPccp) antibody (Fig. 1A). In this case, although no reactivity to full-length TDP-43 was observed under control conditions, TDPccp detected the predicted caspase-cleavage fragment ( $25 \mathrm{kDa})$ as well as several high molecular weight species of TDP-43 following incubation with caspase-3 (Fig. 1A, lane labeled "Digest Casp-3"). To extend these findings, an in vitro model of apoptosis was employed consisting of HeLa cells. HeLa cells were incubated in the presence of the apoptotic insult, SST, for $24 \mathrm{hrs}$ and cell extracts were analyzed by Western blot utilizing the TDPccp antibody. A single doublet band at $25 \mathrm{kDa}$ corresponding to the predicted molecular weight following cleavage of TDP-43 at DVMD ${ }^{219}$ was apparent following treatment with SST (Fig. 1B). The appearance of this SST-induced cleavage fragment was prevented following pretreatment of HeLa cells with the broad caspase inhibitor, Z-VAD (Fig. 1B, lane marked "SST + Z-VAD). A previous study has also identified a doublet band running at $25 \mathrm{kDa}$ fragment of TDP-43 following treatment of extracts with phosphatase \{Amador-Ortiz, 2007 \#2637\}. This suggests that in addition to proteolytical processing, TDP-43 may also undergo abnormal phosphorylation. It is noteworthy the TDPccp antibody did not react with full-length TDP-43 (Fig. 1B). The presence of full-length TDP-43 in HeLa cells was confirmed following Western blot analysis employing a commercial polyclonal antibody to TDP-43 (Fig. 1C). Interesting, this full-length antibody to TDP-43 was unable to detect the $25 \mathrm{kDa}$ caspase-cleavage fragment following treatment of HeLa cells with SST (Fig. 1C).

Following verification that TDPccp is a specific probe for caspase-cleaved TDP-43, immunohistochemical analysis was performed on post-mortem hippocampal brain sections from AD subjects and age-matched controls. Brightfield immunohistochemical analysis employing the TDPccp antibody revealed infrequent labeling in age-match control subjects (Fig. 2A). In general, diffuse staining in control cases was neuronal and was predominantly confined within the hippocampus proper. In contrast, intense, widespread labeling of the TDPccp antibody was observed in all $\mathrm{AD}$ cases examined. Strong immunolabeling was identified within Hirano bodies (Fig. 2B, arrowheads) and these structures were found exclusively within the hippocampus proper (Fig. 2C). Hirano bodies are rod-shaped, cytoplasmic inclusions that are found predominantly within the hippocampus in a variety of neurodegenerative diseases, including AD \{Hirano, 1994 \#2677\}. Labeling of Hirano bodies was also a major finding in two cases neuropathologically diagnosed as being AD/PD (data not shown). It is noteworthy that a previous study identified caspase-cleaved actin within Hirano bodies of AD subjects and revealed a similar staining pattern to what was observed with the TDPccp antibody in the present study \{Rossiter, 2000 \#2676\}. 
Another prominent feature found in AD cases was the extensive labeling of TDPccp within plaque-rich regions (Fig. $2 \mathrm{D}$ and $\mathrm{E}$ ). Labeled plaques were found throughout the hippocampus and were also identified in the entorhinal cortex. TDPccp immunoreactivity was also identified within reactive astrocytes (Fig. 2B, arrows) and within neurons with apparent tangle morphology (Fig. 2F, arrows).

Specificity of the TDPccp antibody as a specific probe for caspase-cleaved TDP-43 in AD was confirmed following experiments with preimmune serum and preadsorbed antibody (Fig. 3). In this manner, there was a complete lack of specific staining in serial AD sections in which preimmune sera was utilized, although nonspecific staining of blood vessels was evident (Fig. 3C). Staining with TDPccp was prevented under conditions whereby purified TDPccp was preadsorbed with the peptide used as the immunogen (Fig. 3F).

Double-label immunofluorescence experiments were undertaken to examine a possible relationship between caspase-cleaved TDP-43 and caspase-cleaved tau. To identify caspase-cleaved tau within tangles, a monoclonal antibody (TauC3) developed by Gamblin et al. was employed, which is specific for the C-terminal caspase-cleavage of tau at aspartic acid 421 \{Gamblin, $2003 \# 694$ \}. Co-localization of both antibodies within neurons was evident in the hippocampus of all AD cases examined (Fig. 4A-C). In addition, we were able to demonstrate the colocalization of TauC3 and TDPccp within dystrophic neurites in plaque regions of the hippocampus (Fig. 4C and D). The concurrence of these two antibodies within neurons and neuritic plaques confirms the specificity of the TDPccp antibody as a marker for caspase-cleaved TDP-43. Finally, co-localization experiments with an anti-A $\beta$ antibody and an antibody to GFAP confirmed the presence of TDPccp within plaque-rich regions as well as within reactive astrocytes of the AD brain (Fig. 4E and F). Previous studies have demonstrated the activation of caspases within reactive astrocytes and the occurrence of caspase-cleaved glial fibrillary acidic protein in the AD brain \{Mouser, $2006 \# 2532\}$.

Because previous studies have shown that pathologic TDP-43 is not only cleaved to generate C-terminal fragments, but is also ubiquinated in affected brain and spinal cord of FTLD-U and ALS \{Kwong, 2007 \#2639\}, double immunohistochemical experiments were performed with the TDPccp antibody and a monoclonal antibody to ubiquitin. Evidence for co-localization of these two markers was apparent within dystrophic neurites (Fig. 5A and C), as well as within neurons with apparent tangle morphology (Fig. 5B and D). The presence of caspase-cleaved TDP-43 together with ubiquinated-positive structures suggests that as in FTLD-U and ALS, pathological TDP-43 undergoes similar turnover events in the AD brain.

In support of our findings, previous studies have shown the occurrence of pathological TDP-43 in a minority of AD cases examined \{Amador-Ortiz, 2007 \#2637; Higashi, 2007 \#2642\}. For both studies, the same commercial antibody to full-length TDP-43 was used (polyclonal, rabbit, ProteinTech, USA). Thus, immunohistochemical analysis was carried out using this antibody and a similar monoclonal antibody to TDP-43 from the same company. Figure 6 depicts the results of experiments utilizing these two antibodies to full-length TDP-43. In general, we found widespread neuronal labeling throughout the hippocampus following application of either of these two antibodies. The staining appeared to be primarily nuclear, and we were unable to identify any pathological structures labeled with the monoclonal antibody to TDP-43 in the majority of cases examined. In addition to nuclear labeling of TDP-43, the polyclonal antibody to TDP-43 immunolabeled dystrophic neurites as well as structures within plaque regions in one $\mathrm{AD}$ case out of five, which corresponds to the frequency of pathological TDP-43 found in a previous study \{Amador-Ortiz, 2007 \#2637\} (Fig. 6C and D).

\section{Discussion}

In the present study, caspase-cleaved TDP-43 has been identified as a major pathological finding in the hippocampus of the AD brain. The identification of caspase-cleaved TDP-43 in AD was accomplished using a caspase-cleavage site-directed antibody to TDP-43 based upon a known caspase-cleavage site at position 219. Cleavage at this site by caspase- 3 would generate a predicted $25 \mathrm{kDa}$ fragment of TDP-43, which has been identified as a major molecular weight species in brain extracts of ALS and FTLD-U \{Amador-Ortiz, 2007 \#2637; Neumann, 2006 \#2634; Zhang, 2007 \#2635\}. Validation of this antibody, termed the TDP caspase-cleavage product (CCP) antibody consisted of several observations: 1) TDPccp immunolabeled a band at $25 \mathrm{kDa}$ following cell-free caspase3 digestion of TDP-43 or following treatment of HeLa cells with the apoptotic insult, SST. The presence of this 25 $\mathrm{kDa}$ band in SST-treated cells was completely prevented following pretreatment with the caspase inhibitor, Z-VAD; 2) TDPccp did not react with full-length TDP-43, whereas under similar experimental conditions an antibody to full- 
length TDP-43 did; 3) TDPccp labeled neurons displayed a cytoplasmic distribution that is characteristic of pathological TDP-43 \{Kwong, 2007 \#2639\}; 4) Staining of TDPccp in the AD sections was completely prevented following preadsorption of the affinity-purified antibody with free peptide or utilizing preimmune serum; 5) a protein-protein BLAST alignment of the seven amino acid residues chosen as the immunogen, QYGDVMD, lists TDP-43 as the only predicted human protein. Taken together, these data support the notion that TDPccp is a specific marker for caspase-cleaved TDP-43.

Application of TDPccp in AD postmortem hippocampal brain sections revealed labeling of Hirano bodies, neuritic plaques, neurons with apparent tangle morphology and reactive astrocytes. Due to the extent and frequency of pathology uncovered following application of this caspase-cleaved TDP-43 antibody, it is proposed that cleaved TDP-43 may represent a major disease component in AD. These results provide further support for a role of apoptosis in general, and caspases in particular in the disease progression associated with AD. Numerous studies have documented caspase activation and cleavage of proteins in the AD brain \{LeBlanc, 2005 \#2581\}. Caspasecleaved TDP-43 can be added to the already extensive list of proteins demonstrated to be substrates for caspases in Alzheimer's including APP \{Gervais, 1999 \#717\}, actin \{Yang, 1998 \#2411\}, fodrin \{Rohn, $2001 \# 1799$ \} and tau \{Rissman, 2004 \#2563; Gamblin, 2003 \#694\}. Recent evidence suggests that caspases may play an early proximal role in promoting the pathology underlying AD including the formation of NFTs and processing of APP and tau \{Spires-Jones, 2008 \#2666; Rohn, 2008 \#2644\}. However, an important caveat to these studies has been the lack of evidence for apoptosis in the AD brain and the role for apoptosis in the AD remains a controversial hypothesis \{Jellinger, $2006 \# 2687$ \}. One possibility is the activation of caspases and the full completion of the apoptotic program and eventual cell death may be largely separated in time \{Rohn, $2002 \# 1808\}$.

Previous studies have demonstrated the presence of pathological TDP-43 in the AD brain \{Amador-Ortiz, 2007 \#2637; Higashi, 2007 \#2642\}. In one study, the authors found TDP-43-positive inclusions in roughly 33\% of the AD cases examined with TDP-43 immunoreactivity being found principally within NCIs but also within dystrophic neurites \{Higashi, 2007 \#2642\}. In another exhaustive study by Amador-Ortiz et al., the authors examined 167 cases of $\mathrm{AD}$ and found pathological TDP-43 in the form of dystrophic neurites and within occasional NFTs in approximately $20 \%$ of the cases examined \{Amador-Ortiz, 2007 \#2637\}. Of interest was the fact that in both studies the same commercial antibody to full-length TDP-43 was utilized (a rabbit polyclonal antibody from Proteintech Group, Inc, USA). Based on previous studies, it appears this antibody reacts with the C-terminal region of TDP-43 \{Neumann, $2006 \# 2634$ \}. The ability of this full-length TDP-43 polyclonal antibody to detect the 25 $\mathrm{kDa}$ caspase-cleaved fragment in an in vitro model system of apoptosis was tested. Although the antibody readily recognized full-length TDP-43 under these conditions, it did not immunolabel the $25 \mathrm{kDa}$ caspase-cleaved fragment of TDP-43 (Fig. 1B). Further, immunohistochemical analysis utilizing this commercial TDP-43 antibody indicated labeling within plaques, apparent NFTs and dystrophic neurites in a minority of AD cases examined. However, unlike TDPccp, this commercial polyclonal antibody to TDP-43 did not label Hirano bodies in the AD brain. These data suggest the difference observed in the staining between these two antibodies may reflect the fact the TDPccp in contrast to the full-length polyclonal TDP-43 was designed to recognize the N-terminal upstream fragment of TDP43, which may be more stable or resistant to turnover than the C-terminal fragment. Thus, in AD following caspase cleavage, the $\mathrm{N}$-terminal fragment of TDP-43 may persist and accumulate allowing for a more consistent detection following immunohistochemical analysis with the TDPccp antibody.

The ability of caspases to cleave TDP-43 may convert TDP-43 to a pathological form allowing for its redistribution to the cytoplasm as well as abnormal accumulation. Biochemical studies of TDP-43 have indicated the presence of ubiquinated high-molecular weight species and C-terminal fragments of TDP-43 in FTLD-U, ALS and the AD brain \{Neumann, 2006 \#2634; Amador-Ortiz, 2007 \#2637\}. It is interesting to speculate whether proteolytic cleavage of TDP-43 enhances its ability to self-aggregate as has been shown for other proteins such as alpha-synuclein \{Dufty, $2007 \# 2643\}$. Whether the caspase-cleavage of TDP-43 facilitates the formation of aggregated species and to what extent these species are neurotoxic will require further investigation.

In conclusion, caspase-cleaved TDP-43 has been identified as a major pathological finding in the AD brain. The presence of caspase-cleaved TDP-43 adds to the long list of substrates demonstrated to be targets for proteolytic cleavage by this family of enzymes in the AD brain. Future studies are needed to determine whether caspase cleavage of TDP-43 is an early or late event, is positively associated with other pathological features of AD, and whether pathological forms of TDP-43 contribute to the neurodegeneration associated with this disease. 


\section{Experimental Procedure}

Materials. The mouse anti-GFAP antibody (MAB3402), mouse TauC3 antibody (caspase-cleaved tau antibody), anti-A $\beta$ mAb 1560 (clone 6E10), and ubiquitin (MAB1510: monoclonal, mouse) was purchased from Chemicon International (Temecula, CA). Two antibodies to full-length (FL) TDP-43 were purchased from Proteintech Group,

Inc.: polyclonal rabbit TARDBP (10782-2-AP) and a mouse monoclonal TARDBP antibody (60019-2-Ig). The beta-actin rabbit polyclonal antibody was from AbCam (Cambridge, MA). Z-Val-Ala-Ala-Asp (OMe)-FMK (ZVAD) was from Enzyme Systems Products (Livermore, CA). Staurosporine (SST) was purchased from Calbiochem (La Jolla, CA).

Generation of the polyclonal caspase-cleaved TDP-43 antibody. Polyclonal antibodies were synthesized based upon a putative caspase cleavage consensus site $\left(\mathrm{DVMD}^{219}\right)$ within TDP-43. We chose the peptide CQYGDVMD, which represents the amino-terminal upstream neoepitope fragment of TDP-43 that would be generated following cleavage by caspases. Following synthesis, this peptide was coupled to KLH and injected into rabbits. The resulting sera (verified by ELISAs) were used to affinity purify antibodies using a sulfolink column coupled with the peptide CQYGDVMD. For this antibody, synthesis of peptides, injections of immunogens, and collection of antisera were contracted out to Bethyl laboratories (Montgomery, TX).

\section{Cell Culture}

Human HeLa cells (ATCC, Manassas, VA) were grown in Eagle's Minimal Essential medium (ATCC, \#30-2003) supplemented with $10 \%$ fetal bovine serum, $100 \mathrm{U} / \mathrm{ml}$ penicillin and $100 \mu \mathrm{g} / \mathrm{ml}$ streptomycin. To initiate apoptosis, cells were treated for $24 \mathrm{hr}$ with the apoptotic insult staurosporine (SST) in serum-free media. SST was made up as a $5 \mathrm{mM}$ stock in sterile DMSO and diluted 1:100 in BSA/PBS before addition to cell cultures. Experiments were also carried out in the presence of the pan caspase inhibitor Z-VAD. Z-VAD was prepared as a $50 \mathrm{mM}$ stock in sterile DMSO. To permit adequate cellular loading, Z-VAD was added 1 hour before insult.

\section{Cell-free digestion of TDP-43}

To examine whether caspase- 3 can cleave TDP-43, $20 \mu \mathrm{g}$ of purified human recombinant TDP-43 was incubated with active human recombinant caspase- 3 in $2 \mathrm{x}$ reaction buffer containing $10 \mathrm{mM}$ DTT for two hours at $37^{\circ} \mathrm{C}$. Reactions were terminated by the addition of $5 \mathrm{x}$ sample buffer and stored at $-20^{\circ} \mathrm{C}$ until analyzed.

\section{Western blot analysis}

HeLa cell extracts were processed for Western blot analysis. Proteins were separated by $12 \%$ SDS-PAGE and transferred to nitrocellulose. Transferred slabs were stained in coomassie blue to verify equal loading between samples. Membranes were incubated in the TDP-43 caspase-cleavage product antibody (TDPccp, 1:500) or rabbit polyclonal FL TDP-43 (Proteintech, 1:500) and primary antibodies were visualized using goat anti-rabbit HRPlinked secondary (1:5,000; Jackson's Laboratory, West Grove, PA), followed by ECL detection. All samples were analyzed for protein content using the BCA assay (Pierce) to ensure equal protein loading.

\section{Human Subjects}

Autopsy brain tissue from the hippocampus and entorhinal cortex of seven neuropathologically confirmed AD cases and five nondemented cases diagnosed as normal was studied. In addition, two cases neuropathologically designated as AD/Parkinson's (PD) were also studied. Human brain tissues used in this study were provided by the Institute for Brain Aging and Dementia Tissue Repositories at the University of California, Irvine.

\section{Immunohistochemistry and Immunofluorescence Microscopy}

Free-floating $40 \mu \mathrm{m}$-thick serial sections were used for immunohistochemical and immunofluorescence studies as previously described \{Rohn, $2002 \# 1807\}$. Antibody dilutions were the following: TDPccp (1:100), GFAP (1:400), anti beta-amyloid mAb 1560 clone 6E10 (1:400), FL TDP-43 (mouse and polyclonal, 1:100) and mAb TauC3 (1:100). To visualize beta-amyloid staining, sections were pretreated for 7 minutes in $95 \%$ formic acid. Antigen visualization was determined using $\mathrm{ABC}$ complex ( $\mathrm{ABC}$ Elite immunoperoxidase kit, Vector labs), followed by DAB substrate (Vector Labs). For immunofluorescence co-localization studies, antigen visualization was accomplished using an Alexa fluor 488-labeled tyramide (green, Ex/Em = 495/519) or streptavidin Alexa Fluor 555 $($ red, Ex/Em $=555 / 565)$, both from Invitrogen $($ Carlsbad, CA). 
Acknowledgments: The authors would like to thank Andrew Curtright, Carson Kidwell and Christopher Anderson for their technical assistance. Funded by NIH/NCRR grant \#P20RR016454 and a grant from the American Health Assistance Foundation (AHAF).

\section{Abbreviations:}

AD- Alzheimer's disease

A $\beta$ - beta amyloid

CCPs- caspase-cleavage products

FTLD-U frontaltemporal lobar degeneration with ubiquitin-positive inclusions

GFAP- glial fibrillary acidic protein

NFTs- neurofibrillary tangles

SST-Staurosporine

TDP-43- TAR DNA-binding protein-43

TDPccp-TDP-43 caspase cleavage product antibody

Figure 1. Characterization of TDPccp antibody by Western blot analysis.

(A): Recombinant human TDP-43 was incubated in the presence or absence of caspase- 3 for 2 hours at $37^{\circ} \mathrm{C}$. Samples were separated by SDS-PAGE, transferred to nitrocellulose and probed with affinity-purified TDPccp antibody (1:500). Western blot analysis indicated that the TDPccp antibody recognizes a principle $25 \mathrm{kDa}$ fragment following digestion with caspase-3. (B): Cell extracts from Hela cells treated with $1 \mu \mathrm{M}$ staurosporine (SST) were run on $12 \%$ SDS-PAGE gels for Western analysis and were probed with purified TDPccp. A prominent doublet band at $25 \mathrm{kDa}$ was labeled that was absent after pretreatment of Hela cells with the caspase inhibitor, Z-VAD. No reactivity to full-length TDP-43 was observed (lane marked control, Panel B). (C): Identical experimental set-up in this case samples were probed with a commercially available antibody to TDP-43, which recognizes full-length TDP-43. This antibody recognized full-length TDP-43, but did not immunolabel the $25 \mathrm{kDa}$ caspase-cleaved fragment full-length TDP-43, but did not immunolabel the $25 \mathrm{kDa}$ caspase-cleaved fragment (B).

Figure 2. Detection of caspase-cleaved TDP-43 in the hippocampus of the Alzheimer's disease brain.

(A): Representative age-matched control case with affinity-purified TDPccp (1:100) showing weak labeling within neurons of the hippocampus. (B-F): Representative staining with TDPccp in AD cases illustrating widespread labeling of the antibody within Hirano bodies (arrowheads, B and C), reactive astrocytes (arrows, B), within plaquerich regions (low field, D; high field, E), and NFTs (arrows, F). All panels are representative staining in the hippocampus. Scale bars are equivalent to $10 \mu \mathrm{m}$ except panel D, which represents $50 \mu \mathrm{m}$.

Figure 3. Confirmation of specificity of the TDPccp antibody by immunohistochemistry.

(A-C): Serial sections from a representative AD case were incubated with immunized serum (A and B) or preimmune serum (C). Specific staining was only observed following application of immunized serum. (D-F): Serial AD sections were immunolabeled with purified TDPccp (1:200) and labeling of Hirano bodies (B), dystrophic neurites (arrowhead, D), plaques (arrow, D), or reactive astrocytes (E) was observed. In contrast, staining with purified TDPccp was prevented after preadsorption with free peptide $(\mathrm{F})$. All scale bars represent $10 \mu \mathrm{m}$, except for Panel F, which represents $20 \mu \mathrm{m}$.

Figure 4. Caspase-cleaved TDP-43 co-localizes with caspase-cleaved tau and is evident within astrocytes as well as plaque-rich regions of the Alzheimer's brain. (A-D): Overlap immunofluorescence images using a monoclonal antibody to caspase-cleaved tau (1:100, green) and TDPccp $(1: 100$, red). Yellow, orange coloring represents areas where markers are overlapping. Co-localization of the two antibodies was evident within tanglebearing neurons (A and B) as well as within dystrophic neurites in plaques (arrows, C and D). (E): Overlap immunofluorescence image showing caspase-cleaved TDP-43 in green and beta-amyloid in red (mAb 1560, clone 6E10). Notice in this case, there is no co-localization of the two 
antibodies, indicating that although labeling is occurring in the same region, beta-amyloid is extracellular while caspase-cleaved TDP-43 is intra-neuronal. (F): Double immunofluorescence labeling in reactive astrocytes of the AD brain utilizing a monoclonal antibody to full-length GFAP (green, 1:400) and TDPccp (red, 1:100). Colocalization of the two antibodies was evident within astrocytes of the AD brain. All scale bars represent $10 \mu \mathrm{m}$.

Figure 5. The TDPccp antibody co-localizes with an anti-ubiquitin mAB antibody in the AD brain. Representative double immunostaining with TDPccp (red brown) and ubiquitin (blue gray) in the hippocampus of the AD brain. Note the co-localization of both markers within plaques (arrow, A), neurons with apparent NFT morphology (B and D), and Hirano bodies (arrow, C). Scale bars represent $10 \mu \mathrm{m}$.

Figure 6. Full-length antibodies to TDP-43 reveal widespread nuclear and pathological labeling within the hippocampus of the AD brain. Two different antibodies to full-length TDP-43 were examined by immunohistochemistry utilizing hippocampal brain sections from representative AD cases. Both a commercially available monoclonal antibody to full-length TDP-43 (A and B) or a polyclonal antibody to full-length TDP-43 (C and D) was employed. Application of the monoclonal antibody to FL TDP-43 revealed widespread nuclear labeling within neurons in all hippocampal regions examined (A and B). In contrast, the rabbit polyclonal FL TDP-43 antibody immunolabeled plaques (arrows, C), apparent NFTs (arrows) and dystrophic neurites (arrowheads, D). Scale bars are $100 \mu \mathrm{m}$ for Panel A and $10 \mu \mathrm{m}$ for Panels B, C and D.

\section{References Cited}

1. Amador-Ortiz C, Lin WL, Ahmed Z, Personett D, Davies P, Duara R, Graff-Radford NR, Hutton ML, Dickson DW. TDP-43 immunoreactivity in hippocampal sclerosis and Alzheimer's disease. Ann Neurol. 2007;61:435-445.

2. Buratti E, Brindisi A, Pagani F, Baralle FE. Nuclear factor TDP-43 binds to the polymorphic TG repeats in CFTR intron 8 and causes skipping of exon 9: a functional link with disease penetrance. Am J Hum Genet. 2004;74:13221325

3. Cotman CW, Poon WW, Rissman RA, Blurton-Jones M. The role of caspase cleavage of tau in Alzheimer disease neuropathology. J Neuropathol Exp Neurol. 2005;64:104-112.

4. Dufty BM, Warner LR, Hou ST, Jiang SX, Gomez-Isla T, Leenhouts KM, Oxford JT, Feany MB, Masliah E, Rohn TT. Calpain-cleavage of alpha-synuclein: connecting proteolytic processing to disease-linked aggregation. $\mathrm{Am}$ J Pathol. 2007;170:1725-1738.

5. Gamblin TC, Chen F, Zambrano A, Abraha A, Lagalwar S, Guillozet AL, Lu M, Fu Y, Garcia-Sierra F, LaPointe N, Miller R, Berry RW, Binder LI, Cryns VL. Caspase cleavage of tau: linking amyloid and neurofibrillary tangles in Alzheimer's disease. Proc Natl Acad Sci U S A. 2003;100:10032-10037.

6. Gervais FG, Xu D, Robertson GS, Vaillancourt JP, Zhu Y, Huang J, LeBlanc A, Smith D, Rigby M, Shearman MS, Clarke EE, Zheng H, Van Der Ploeg LH, Ruffolo SC, Thornberry NA, Xanthoudakis S, Zamboni RJ, Roy S, Nicholson DW. Involvement of caspases in proteolytic cleavage of Alzheimer's amyloid- beta precursor protein and amyloidogenic A beta peptide formation. Cell. 1999;97:395-406.

7. Higashi S, Iseki E, Yamamoto R, Minegishi M, Hino H, Fujisawa K, Togo T, Katsuse O, Uchikado H, Furukawa Y, Kosaka K, Arai H. Concurrence of TDP-43, tau and alpha-synuclein pathology in brains of Alzheimer's disease and dementia with Lewy bodies. Brain Res. 2007;1184:284-294.

8. Hirano A. Hirano bodies and related neuronal inclusions. Neuropathol Appl Neurobiol. 1994;20:3-11.

9. Jellinger KA. Challenges in neuronal apoptosis. Curr Alzheimer Res. 2006;3:377-391. 
10. Josephs KA, Whitwell JL, Duffy JR, Vanvoorst WA, Strand EA, Hu WT, Boeve BF, Graff-Radford NR, Parisi JE, Knopman DS, Dickson DW, Jack CR, Jr, Petersen RC. Progressive aphasia secondary to Alzheimer disease vs FTLD pathology. Neurology. 2008;70:25-34.

11. Kwong LK, Neumann M, Sampathu DM, Lee VM, Trojanowski JQ. TDP-43 proteinopathy: the neuropathology underlying major forms of sporadic and familial frontotemporal lobar degeneration and motor neuron disease. Acta Neuropathol. 114;2007:63-70.

12. Kwong LK, Uryu K, Trojanowski JQ, Lee VM. TDP-43 proteinopathies: neurodegenerative protein misfolding diseases without amyloidosis. Neurosignals. 2008;16:41-51.

13. LeBlanc AC. The role of apoptotic pathways in Alzheimer's disease neurodegeneration and cell death. Curr Alzheimer Res. 2005;2:389-402.

14. Mouser PE, Head E, Ha KH, Rohn TT. Caspase-mediated cleavage of glial fibrillary acidic protein within degenerating astrocytes of the Alzheimer's disease brain. Am J Pathol. 2006;168:936-946.

15. Neumann M, Sampathu DM, Kwong LK, Truax AC, Micsenyi MC, Chou TT, Bruce J, Schuck T, Grossman M, Clark CM, McCluskey LF, Miller BL, Masliah E, Mackenzie IR, Feldman H, Feiden W, Kretzschmar HA, Trojanowski JQ, Lee VM. Ubiquitinated TDP-43 in frontotemporal lobar degeneration and amyotrophic lateral sclerosis. Science. 2006;314:130-133.

16. Rissman RA, Poon WW, Blurton-Jones M, Oddo S, Torp R, Vitek MP, LaFerla FM, Rohn TT, Cotman CW. Caspase-cleavage of tau is an early event in Alzheimer disease tangle pathology. J Clin Invest. 2004;114:121-130.

17. Rohn TT, Head E, Nesse WH, Cotman CW, Cribbs DH. Activation of caspase-8 in the Alzheimer's disease brain. Neurobiol Dis. 2001;8:1006-1016.

18. Rohn TT, Head E, Su JH, Anderson AJ, Bahr BA, Cotman CW, Cribbs DH. Correlation between caspase activation and neurofibrillary tangle formation in Alzheimer's disease. Am J Pathol. 2001;158:189-198.

19. Rohn TT, Rissman RA, Davis MC, Kim Y-E, Cotman C, Head E. Caspase-9 Activation and caspase cleavage of tau in the Alzheimer's disease brain. Neurobiol Dis. 2002;11:341-354.

20. Rohn TT, Rissman RA, Head E, Cotman CW. Caspase activation in the Alzheimer's disease brain: tortuous and torturous. Drug News Perspect. 2002;15:549-557.

21. Rohn TT, Vyas V, Hernandez-Estrada T, Nichol KE, Christie LA, Head E. Lack of pathology in a triple transgenic mouse model of Alzheimer's disease after overexpression of the anti-apoptotic protein Bcl-2. J Neurosci. 2008;28:3051-3059.

22. Rossiter JP, Anderson LL, Yang F, Cole GM. Caspase-cleaved actin (fractin) immunolabelling of Hirano bodies. Neuropathol Appl Neurobiol. 2000;26:342-346.

23. Spires-Jones TL, de Calignon A, Matsui T, Zehr C, Pitstick R, Wu HY, Osetek JD, Jones PB, Bacskai BJ, Feany MB, Carlson GA, Ashe KH, Lewis J, Hyman BT. In vivo imaging reveals dissociation between caspase activation and acute neuronal death in tangle-bearing neurons. J Neurosci. 2008;28:862-867.

24. Uryu K, Nakashima-Yasuda H, Forman MS, Kwong LK, Clark CM, Grossman M, Miller BL, Kretzschmar HA, Lee VM, Trojanowski JQ, Neumann M. Concomitant TAR-DNA-binding protein 43 pathology is present in Alzheimer disease and corticobasal degeneration but not in other tauopathies. J Neuropathol Exp Neurol. 2008;67:555-564.

25. Yang F, Sun X, Beech W, Teter B, Wu S, Sigel J, Vinters HV, Frautschy SA, Cole GM. Antibody to caspasecleaved actin detects apoptosis in differentiated neuroblastoma and plaque-associated neurons and microglia in Alzheimer's disease [see comments]. Am J Pathol. 1998;152:379-389. 
26. Zhang YJ, Xu YF, Dickey CA, Buratti E, Baralle F, Bailey R, Pickering-Brown S, Dickson D, Petrucelli L. Progranulin mediates caspase-dependent cleavage of TAR DNA binding protein-43. J Neurosci. 2007;27:1053015534. [PubMed] 